\title{
An AC Controlled-Current Load for Controllable Waveform Parameters to Quantify Static Energy Meter Errors
}

\author{
Johan Dijkstra $^{1}$, Tom Hartman ${ }^{1}$, Niek Moonen ${ }^{1}$, Frank Leferink ${ }^{1,2}$ \\ ${ }^{1}$ University of Twente, Enschede, the Netherlands \\ ${ }^{2}$ THALES Nederland B.V., Hengelo, the Netherlands \\ tom.hartman@utwente.nl
}

\begin{abstract}
This paper proposes a method for quantifying static energy meter errors using current waveform parameters. This will be realized via a controlled-current load, for which the design is given. Several parameters like the rise time, current slope, crest factor and phase firing angle are considered to identify relations which cause static energy meter measurement errors. Some of these parameters are also used as a way to verify the functionality of the controlled-current load in a quantitative manner. This is realized by comparing these parameters for the original and reproduced current waveform, where a feedback capacitor is used to optimize the system tailored to which parameter is deemed most crucial.
\end{abstract}

Index Terms-Static Meter, Energy Meter, Controlled-Current, AC Load, Waveform Parameters

\section{INTRODUCTION}

Nowadays static energy meters are used to replace their mechanical counterparts to measure the energy consumption in modern household situations [1]-[3]. Throughout Europe, utility companies are currently deploying these meters. However, major deviations in some of the static energy meter readings have been reported in recent studies, despite the meters fulfilling the harmonised standards [4]-[7].

Due to the immense impact of the problems with the static energy meters, it is critical that the actual extent is determined. In fact, the MeterEMI European research project is working towards a thorough understanding of the reported static energy metering error problem [8]. The main objective of this project is to develop new standards such that the performance of the meters can be analyzed.

Subsequently, it is necessary to characterize and identify the parameters of these waveforms to determine the cause of the errors. Up until now the appliances, which caused errors on the static energy meters, all exhibited a non-linear way of drawing current. The parameters that are often considered for analyzing these, mostly impulsive, waveforms are the rise time, fall time, current slope $(\mathrm{d} I / \mathrm{d} t)$, crest factor $(\mathrm{CF})$, phase firing angle (FA), power factor (PF), peak current, total harmonic distortion (THD) and the energy (pulse width), for which the current

This project has received funding from the EMPIR programme co-financed by the Participating States and from the European Union's Horizon 2020 research and innovation programme. The results found reflect the author's view only. EURAMET is not responsible for any use that may be made of the information it contains. slope has already been correlated to errors [9]. Consumers complained about a speed-controlled water pump increasing their energy readings, so research has been done on this type of pump in combination with a dimmer. The dimming level changes the current slope, which in turn has an effect on the magnitude of the error. An inductance was added to the system to decrease slope of the current and the change in current slope was correlated to the static energy meter errors. Aside from changing the current slope, in discrete non-linear steps, this does however also change several of the other parameters in an uncontrollable manner. It is therefore necessary to control the amount of current, after which parameters can be adjusted in a controllable practice to quantitatively determine the effect a parameter, or a combination of several parameters, has on the energy measurements.

To realize the aforementioned, an arbitrarily adjustable load is proposed. The commercial of the shelf (COTS) alternating current (AC) loads are generally limited and very expensive [10], [11]. These loads all comply with standards limiting their functionalities, thus extreme current waveforms from real household appliances and custom waveforms, with very high slew rates, cannot be replicated. Limitations of waveforms are usually set by the $\mathrm{CF}$ and $\mathrm{PF}$, where the maximum $\mathrm{CF}$ is 5 for the previously mentioned COTS AC loads. For these reasons, a high performance, arbitrary AC controlled-current load will be designed to test static energy meters.

This paper starts with describing the parameters that will be used to characterize the waveforms in Section II. After this, in Section III, waveforms from household equipment will be analyzed and their parameters will be correlated to some of the errors of the static energy meters. This is followed up by a description of the design of the AC controlled-current load planned to be constructed in Section IV. In Section V the given waveforms will be reproduced by the simulation of the $\mathrm{AC}$ controlled-current load to quantitatively test the design.

\section{WAVEFORM PARAMETERS}

The voltage and current waveforms of appliances will give insight into the root cause of the errors of static energy meters, as these errors can be coupled to the parameters of these waveforms. The appliances which caused erroneous readings all exhibit a non-linear way of drawing current. Therefore it is 
important to determine the effect current waveform parameters have on the energy measurement errors.

\section{A. Definition}

Most appliances tested draw non-linear currents which makes investigating the parameters of the current the main focus. However it is still important to consider some of the voltage parameters, for example the $\mathrm{THD}_{\mathrm{V}}$, which can have an impact on the functionality of an appliance. Other parameters that can be considered, specifically for the current, are: rise time, fall time, current slope $(\mathrm{d} I / \mathrm{d} t), \mathrm{CF}$, phase FA, PF, peak current and the energy (pulse width). It has been shown that the current slope and the phase FA can been correlated to errors of static energy meters [9]. This has been realized by changing the current that is being drawn in discrete steps via the use of a dimmer. However since some parameters are interdependent, this means that changing one parameters may lead to other parameters changing as well. As a result, this makes it impossible to fully determine the effect of a single parameter on the cause of the errors of static energy meters via this uncontrollable way of using a real appliance.

The current slope, or current slew rate, gives an indication of how fast the current rises or falls and the definition for two different waveforms can be seen in Fig. 1. The slew rate is defined as the slope between 10 and $90 \%$ of the pulse, and where the time it takes to reach these levels is considered as the rise time or fall time for rising edges and falling edges respectively.

Furthermore we know that the $\mathrm{CF}$ is the ratio between the peak current and the root mean square (RMS) current, where a larger CF corresponds to a narrower or larger current pulse. The phase FA is defined as the phase between the point where the current starts conducting and the previous zero crossing (ZC) of the voltage waveform and is written down in Eq. 1.

$$
\mathrm{FA}=\frac{t_{\text {pulse }}-t_{\mathrm{ZC}}}{T_{\text {grid }}} \cdot 360^{\circ}
$$

The PF of a non-linear load is determined via the main $50 \mathrm{~Hz}$ component and the harmonics of the current and the voltage. The peak current is defined as the maximum current in the waveform and the energy of the current pulse can be found by integrating the current pulse.
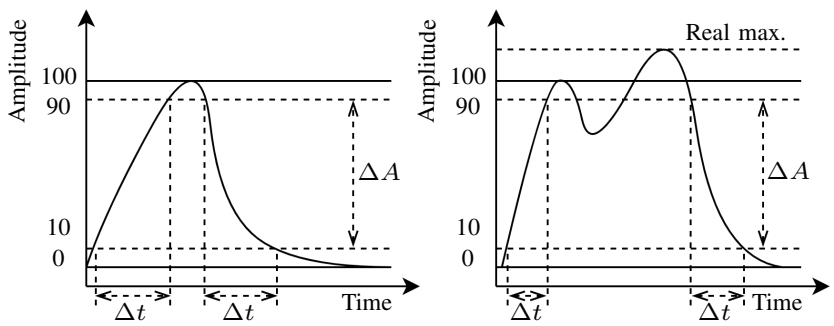

Fig. 1: Definition of current slew rate

\section{B. Error correlation}

To acquire a better understanding of why static energy meters give measurement errors, a quantitative analysis will be done, where parameters will be adjusted in a controllable environment. Ideally one would change exactly one parameter and determine the effect it has on the errors of the static energy meters. However, this is not possible for all parameters, as some are interconnected and dependent on each other. As an example, one cannot only increase the CF without also increasing the power. This fact makes it extremely hard to say something quantifiable about the measurements results when only using COTS appliances, where many parameters change in an uncontrolled manner between measurements.

\section{WaVeform Parameters - Static Energy Meter ERRORS RELATION}

In this paper, a few of the aforementioned parameters will be considered to identify possible relations between these parameters and the errors of static meters. The data has been gathered using a multi-channel digitizer, PicoScope, where the voltage and the current have been measured simultaneously. The parameters have been calculated using MATLAB, which will also be used as a part of the verification of the functionality of the AC controlled-current load.

\section{A. Possible Parameter Relations}

The parameters of certain waveforms can be seen in Table I, where six waveforms have been measured for different dimming settings. Lvl. 1 dimming implies the most dimming of the current waveform, and therefore the lowest power, whereas lvl. 10 implies no dimming. The parameters we will look at are the phase FA, CF, PF versus the difference in calculated power, where the Yokogawa WT500 power analyzer is used as the reference meter for the static energy meter.

At first glance it can be seen from the data that there is a correlation between the $\mathrm{CF}$ and the error in the static energy meter measurements. This finding is not unexpected since there is a big relation between the slew rate of a certain pulse and the $\mathrm{CF}$ of a certain waveform. However this is not a one to one relation since a pulse with a high slew rate can coincide on top of a relatively high sine wave, as the superposition of different appliances. In such a case the signal would have a high slew rate but the $\mathrm{CF}$ would be significantly smaller. These two cases would be interesting to elaborate on to determine the effect of the individual parameters. Next to the CF the PF also seems an interesting parameter because the same trend can be seen, but again one needs to consider the other parameters since the phase FA of the pulse has an impact on the power factor of the waveform.

Just like the effect of the current slew rate, [9] also proposes the effect the phase FA has on the static energy meter errors, just like the correlation that can be seen in the table. It is however important to notice that the error calculation is a percentage with respect to the reference measurement of the Yokogawa WT500. This means that for the same relative error a higher percentage of an error will occur, when the actual 
TABLE I: Parameters and measurement errors for five different waveforms

\begin{tabular}{|c|c|c|c|c|c|c|c|}
\hline Dimming & $I_{\max }[\mathrm{A}]$ & Phase Firing Angle $\left[^{\circ}\right]$ & Crest Factor & Power Factor & Power Yokogawa [W] & Power SM [W] & Power Error [\%] \\
\hline Lvl. 1 & 26.90 & 160.6 & 12.4 & 0.06 & 29.2 & 629.9 & 2054.4 \\
\hline Lvl. 3 & 19.06 & 151.4 & 10.6 & 0.10 & 44.4 & 367.6 & 727.4 \\
\hline Lvl. 5 & 22.61 & 138.5 & 10.9 & 0.13 & 64.3 & 449.9 & 599.1 \\
\hline Lvl. 7 & 19.49 & 121.8 & 9.9 & 0.20 & 90.4 & 265.6 & 193.7 \\
\hline Lvl. 9 & 19.92 & 103.5 & 9.0 & 0.23 & 123.3 & 209.2 & 69.8 \\
\hline
\end{tabular}

power consumption has been dimmed, due to the smaller denominator. For this reason we should also take a look at the actual difference in power, however still no strong conclusion can be made about the effect of the phase FA on the error, as of yet, due to the fact that other parameters are also changing in the current setup.

This data and these conclusions are used to identify possible relations. It should be clear that no real relation can be given with this limited data set and the variation in all the parameters between measurements. Exactly for this reason the AC controlled-current load is proposed in this paper to realize a controllable lab setup to reliably focus on certain parameters at a time and at different combinations of them.

\section{Design of An AC Controlled-Current LoAd}

The specifications of the AC controlled-current load are based on the waveforms of existing appliances, since the load must be able to, at least, replicate these waveforms. A basic direct current (DC) controlled-current load will be used as a starting point and adaptations will be made to add AC functionality. Afterwards, the external effect of the parasitic inductance will be discussed, as this has a great impact on the current slew rate.

\section{A. Specifications}

The load must be able to replicate current waveforms of existing appliances, as well as arbitrary, custom waveforms. The load will be able to control the current, i.e. controlledcurrent. A set of 61 different measurement data has been used as a basis for the limitations for the design, where a MATLAB script extracted the worst case scenario of some of the previously mentioned parameters. These measurements are from appliances like water pumps, heaters or compact fluorescent lamp (CFL) and light-emmiting diode (LED) lamps in several combinations with dimmers. A margin of at least $20 \%$ has been added on top of these maxima to give the specifications of the AC load, given in Table II. Only waveforms which gave erroneous static energy meter readings have been considered for determining the minimum requirements of the AC load, but the overdimensioned specifications are broad enough to replicate any waveform which complies with these specifications. As will be shown later, the current slew rate is mainly dependent on the impedance of the AC voltage source, thus this requirement only holds when the load is attached to a source with a low impedance, which explains the high slew rate. The load will only be able to sink the current, which limits the operation to the first and third quadrant where the voltage and current have the same sign.
TABLE II: AC Controlled-current load specifications

\begin{tabular}{|l|l|}
\hline Operating voltage & $3.5-500 \mathrm{~V}$ \\
\hline Current range & $0-25 \mathrm{~A}$ \\
\hline Nominal power & $1 \mathrm{~kW}$ \\
\hline Peak power & $6 \mathrm{~kW}$ \\
\hline Current slew rate & $15 \mathrm{~A} / \mu \mathrm{s}$ minimal \\
\hline
\end{tabular}

\section{B. Basic Controlled-Current Load circuit}

A basic controlled-current DC load circuit, given in Fig. 2, has been used as the basis for the design. It consists of a metal-oxide-semiconductor field-effect transistor (MOSFET), current shunt resistor and an operational amplifier (op-amp). The MOSFET is used as a variable resistor and is driven by the op-amp, such that the voltage over the current shunt is equal to the reference voltage. However, this circuit alone will not operate for $\mathrm{AC}$, as a negative voltage causes a short due to the intrinsic body diode of the MOSFET. Next to that, the current waveform needs to be synchronized with the sinusoidal voltage. Thus, adaptations have been made to make it operate with an $\mathrm{AC}$ voltage.

\section{Adaptations}

To convert the AC voltage to a positive voltage, a diode bridge has been added at the input of the basic controlledcurrent load circuit, as can be seen in block A in Fig. 3. A smoothing capacitor has not been added, as this would take away the controllability of the current. The diode bridge introduces a voltage drop, which limits the operation around ZCs. The current draw around ZCs is also limited, due to the

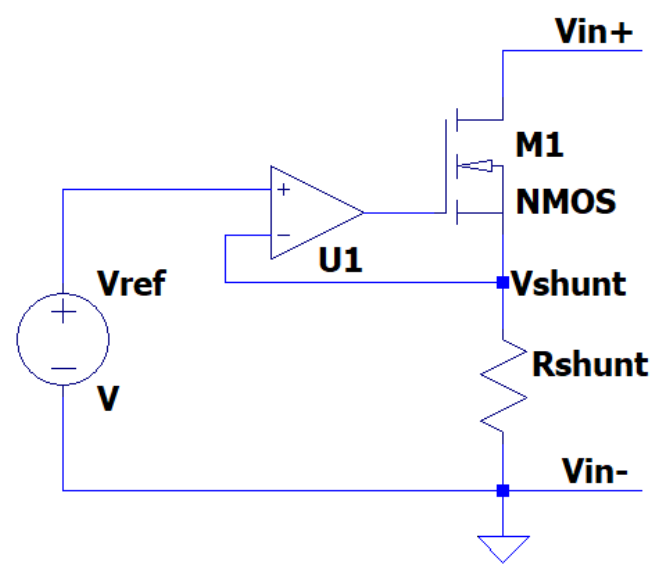

Fig. 2: Basic controlled-current DC load circuit 


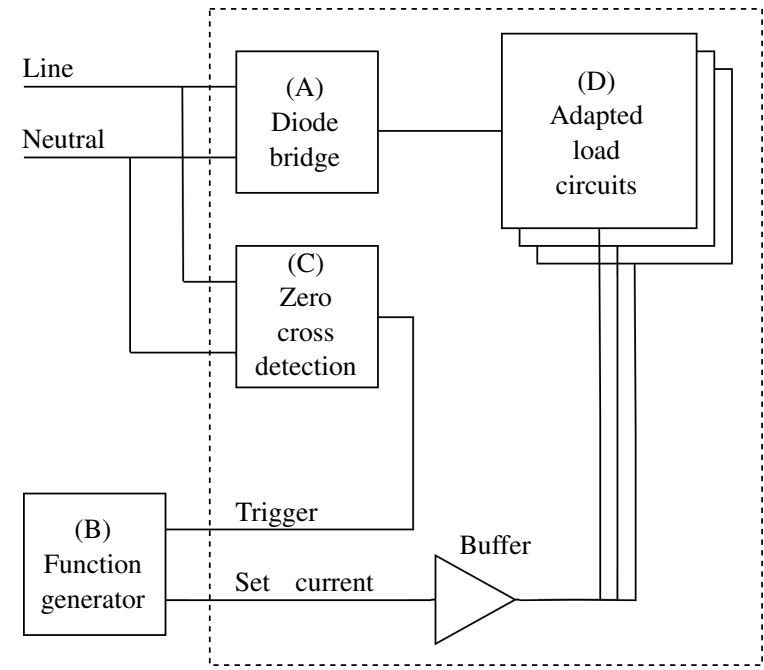

Fig. 3: Block diagram of an AC controlled-current load

additional voltage drop of the current shunt and MOSFET, which is dependent on the magnitude of the current. Therefore, the load will be disabled when the voltage is less than $3.5 \mathrm{~V}$ to prevent current flow at relatively low voltages.

The current waveform will be generated by a function generator, given as block B in Fig. 3, which will output a signal which is exactly one period long. This will allow for a different current waveform in the positive and negative halves of the sinewave period. The function generator is triggered by a $\mathrm{ZC}$ detection circuit, block $\mathrm{C}$, which gives a pulse when the $\mathrm{AC}$ voltage goes through zero, and has a positive slope. A comparator with a Schmitt trigger prevents erroneous triggering caused by noise, which creates multiple ZCs.

A shunt resistor is used to convert the current to a voltage, but incorrect readings might occur when parasitics are present in the ground connections. These parasitics will raise the local ground potential, which is dependent on the current, and this will result in a higher and incorrect current reading. This problem is solved when the voltage over the shunt is measured differentially, which is done via a differential amplifier.

The MOSFET is biased to make it conduct a small amount of current through the drain. This increases the responsiveness of the circuit, as this bias results in the gate-source voltage being higher than the threshold voltage. Next to that, less charge needs to be inserted for the same change in current, as the bias results in the input capacitance of the MOSFET being partly charged. This lowers the output current requirement of the op-amp when a large current slope needs to be replicated.

Instability is inherently present inside the circuit and is caused by the input capacitance of the MOSFET and output impedance of the op-amp. Next to that, a higher AC voltage source impedance also increases instability. Hence, a feedback capacitor has been added between the output of the op-amp and its inverting input to increase the stability of the circuit.

These adapted AC controlled-current load circuits are placed in parallel such that the current and power are shared among multiple transistors, visualized in block D. This eases the minimum current slew rate, maximum current and power requirements and allows for a higher shunt resistance. MOSFETs with lower specifications can also be selected, as the requirements per transistor become more lenient.

\section{Effect of parasitic inductance}

Next to the limitations around ZCs, due to the voltage drop, the current slew rate around ZCs is also limited. Lower voltages and an $\mathrm{AC}$ voltage source with a relatively high impedance will prevent a fast change in current. For this reason it is mandatory that a source with a low impedance is used, when waveforms with a relatively high current slew rate need to be replicated. This fact is especially true with current pulses around the $\mathrm{ZC}$ of the voltage. When the external impedance is modeled as a resistor-inductor (RL) circuit, where an inductor is added in series with the AC load, an expression can be made for the maximum slew rate as;

$$
\frac{\mathrm{d} I_{\text {shunt }}}{\mathrm{d} t}=\frac{0.39 I_{\max }}{0.5 \tau}=\frac{0.78 \frac{V_{\text {source }}}{R_{\text {eq }}}}{\frac{L_{\text {parasic }}}{R_{\text {eq }}}}=\frac{0.78 V_{\text {source }}}{L_{\text {parasitic }}}
$$

The maximum slope is achieved in the first half of the time constant and where $R_{\text {eq }}$ is the sum of the parasitic, shunt and MOSFET resistance. It can be seen that the current slew rate is dependent on the source voltage and parasitic inductance, where a low voltage and high inductance limit the slew rate.

\section{VerificATION}

The design is simulated in LTspice as a verification. Ten AC controlled-current load circuits have been placed in parallel and each transistor has been biased at $5 \mathrm{~mA}$, giving a standard current draw of $50 \mathrm{~mA}$. An inductor and resistor have been added $(1 \mu \mathrm{H}$ and $0.1 \Omega$ respectively) in series with the AC voltage source to imitate the lab setup. Some of the measurements have been performed using a four-quadrant amplifier with a capacitor between line and neutral (before the static energy meters) to create a low impedance, thus a sufficiently low inductance was chosen to achieve the required current slew rate. A higher impedance will act as a low-pass filter (LPF), which prevents the imitation of current waveforms with relatively high frequency components. Two waveforms have been simulated, where the error between waveform parameters will be used to analyse the performance of the circuit. Different feedback capacitor values will be chosen, as this capacitance determines the response of the circuit. A lower capacitance decreases stability, whereas a higher capacitance introduces a delay and lowers the bandwidth. An optimal capacitor value can be chosen tailored to which parameter is deemed most crucial.

\section{A. Test waveforms}

Two waveforms of appliances have been used for the simulation: one has a high current slew rate of $12.5 \mathrm{~A} / \mu$ s and $-8.85 \mathrm{~A} / \mu \mathrm{s}$, and the other has a relatively high current draw around ZCs, which will verify the performance of the design at low voltages. Both are waveforms from different water pumps and are given in Fig. 4 and Fig. 5 respectively. 


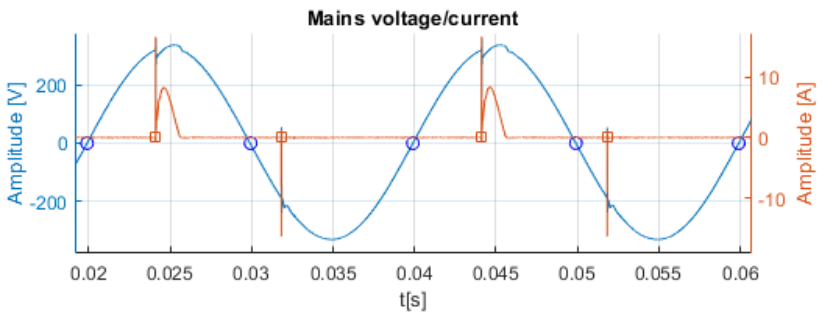

Fig. 4: Waveforms of a water pump in series with a dimmer

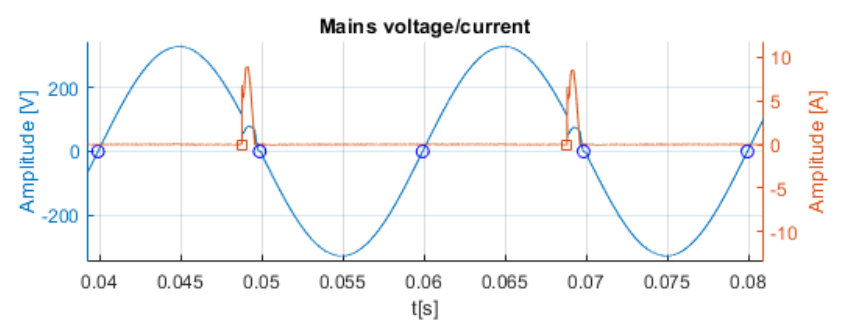

Fig. 5: Waveforms of a remote control water pump

\section{B. Error analysis}

The error between the parameters of the original and replicated waveforms is determined using the same MATLAB script. Important parameters are given in Table III and IV, where errors smaller than $1 \%$ are given in green, errors larger than $2.5 \%$ in red and yellow in between. These tables show that the second waveform has a smaller error for each parameter, showing that it is easier to replicate. The main reason for this is that this waveform does not change as fast compared to the first waveform, i.e. its bandwidth is lower. For the first waveform, there is an optimum between $140 \mathrm{pF}$ and $160 \mathrm{pF}$ where the current slew rate error is minimized. The fall time error drastically increases with capacitance, indicating that the circuit is less responsive at relatively large negative current slew rates in combination with a large feedback capacitor. In general, the errors are larger when compared to the other waveform, as this waveform has a higher $d I / d t$, i.e. higher frequency components. This mainly has to do with the filtering effect of the parasitic inductance, which acts as a LPF. Thus, care must be taken when high current slew rate waveforms are drawn.

\section{CONCLUSION}

This paper proposes a design of an AC controlled-current load to realize a measurement setup to properly determine the relation between certain waveform parameters like the rise time, fall time, current slope $(\mathrm{d} I / \mathrm{d} t), \mathrm{CF}$, phase FA, PF, peak current, THD and the energy (pulse width), and the cause of erroneous measurements of static energy meters. This is realized by slightly adjusting certain parameters in a controllable setup to investigate the effect it has on the magnitude of the error.

Results from a COTS appliance are also given from which certain relations between current waveform parameters and the errors on static energy meters can be identified. However, since a COTS appliance has been used, there was no direct control over the waveform parameters, as multiple parameters changed in an uncontrollable manner. Therefore an $\mathrm{AC}$ controlled-current load was proposed by which custom current waveforms can be drawn from mains, such that waveform parameters can be adjusted precisely. This direct control on the parameters enables the study of its effect on static energy meter measurement errors.

Some of these previously mentioned parameters are also used to quantitatively verify the functionality of the AC controlled-current load. This is done by comparing certain waveform parameters of the reproduced current waveform with the test waveform itself. By adjusting the feedback capacitor in the AC controlled-current load, it has been shown that the error for certain parameters can be minimized. The design has been verified to work within the simulation environment and will be built in the near future to further study the erroneous measurements of static energy meters. 
TABLE III: Percentage errors vs feedback capacitance for the replicated current waveform given in Fig. 4

\begin{tabular}{|c|c|c|c|c|c|c|c|c|c|c|c|c|c|c|c|c|c|c|c|c|}
\hline & $60 \mathrm{pF}$ & $70 \mathrm{pF}$ & $80 \mathrm{pF}$ & $90 \mathrm{pF}$ & $100 \mathrm{pF}$ & 110pF & $120 \mathrm{pF}$ & $130 \mathrm{pF}$ & $140 \mathrm{pF}$ & $150 \mathrm{pF}$ & $160 \mathrm{pF}$ & $170 \mathrm{pF}$ & $180 \mathrm{pF}$ & $190 \mathrm{pF}$ & $200 \mathrm{pF}$ & $210 \mathrm{pF}$ & $220 \mathrm{pF}$ & $230 \mathrm{pF}$ & $240 \mathrm{pF}$ & $250 \mathrm{pF}$ \\
\hline Rise time error & -1.53 & -2.10 & -2.66 & -2.96 & -3.10 & -3.14 & -2.83 & -2.59 & -2.39 & -2.29 & -1.57 & -0.83 & -0.25 & 0.28 & 0.49 & 0.65 & 0.74 & 0.79 & 1.21 & 1.63 \\
\hline Fall time error & 3.15 & 2.76 & 2.55 & 2.54 & 2.59 & 2.70 & 2.85 & 3.03 & 3.25 & 3.49 & 3.74 & 4.03 & 4.33 & 4.66 & 4.99 & 5.35 & 5.71 & 6.07 & 6.46 & 6.86 \\
\hline Slew rate error & 2.86 & 2.55 & 2.52 & 2.39 & 2.20 & 1.98 & 1.44 & 1.02 & 0.67 & 0.45 & -0.39 & -1.21 & -1.87 & -2.42 & -2.66 & -2.85 & -2.96 & -3.03 & -3.46 & -3.88 \\
\hline Max current error & 1.29 & 0.40 & -0.20 & -0.64 & -0.96 & -1.22 & -1.43 & -1.60 & -1.74 & -1.85 & -1.95 & -2.04 & -2.11 & -2.15 & -2.18 & -2.21 & -2.24 & -2.27 & -2.29 & -2.31 \\
\hline Min pulse width error & -0.48 & -0.41 & -0.32 & -0.24 & -0.16 & -0.08 & -0.01 & 0.07 & 0.17 & 0.26 & 0.36 & 0.45 & 0.54 & 0.64 & 0.73 & 0.82 & 0.93 & 1.02 & 1.15 & 1.28 \\
\hline Max pulse width error & 0.35 & 0.35 & 0.37 & 0.34 & 0.34 & 0.34 & 0.36 & 0.36 & 0.36 & 0.36 & 0.36 & 0.35 & 0.35 & 0.36 & 0.35 & 0.35 & 0.33 & 0.35 & 0.35 & 0.35 \\
\hline
\end{tabular}

TABLE IV: Percentage errors vs feedback capacitance for the replicated current waveform given in Fig. 5

\begin{tabular}{|c|c|c|c|c|c|c|c|c|c|c|c|c|c|c|c|c|c|c|c|c|}
\hline & $60 \mathrm{pF}$ & $70 \mathrm{pF}$ & $80 \mathrm{pF}$ & $90 \mathrm{pF}$ & $100 \mathrm{pF}$ & $110 \mathrm{pF}$ & $120 \mathrm{pF}$ & $130 \mathrm{pF}$ & $140 \mathrm{pF}$ & $150 \mathrm{pF}$ & $160 \mathrm{pF}$ & $170 \mathrm{pF}$ & $180 \mathrm{pF}$ & 190pF & $200 \mathrm{pF}$ & $210 \mathrm{pF}$ & $220 \mathrm{pF}$ & $230 \mathrm{pF}$ & $240 \mathrm{pF}$ & $250 \mathrm{pF}$ \\
\hline Rise time error & 2.93 & -0.02 & 0.03 & -0.04 & -0.06 & -0.08 & -0.18 & -0.19 & -0.11 & -0.12 & -0.24 & -0.25 & -0.25 & -0.26 & -0.21 & -0.22 & -0.22 & -0.23 & -0.24 & -0.24 \\
\hline Fall time error & -0.84 & 0.15 & 0.50 & 0.55 & 0.55 & 0.55 & 0.55 & 0.56 & 0.56 & 0.57 & 0.57 & 0.60 & 0.59 & 0.76 & 0.60 & 0.62 & 0.62 & 0.63 & 0.64 & 0.64 \\
\hline Slew rate error & -0.58 & 0.53 & 0.45 & 0.48 & 0.47 & 0.46 & 0.55 & 0.55 & 0.46 & 0.46 & 0.57 & 0.58 & 0.58 & 0.58 & 0.53 & 0.53 & 0.54 & 0.54 & 0.54 & 0.55 \\
\hline Max current error & 2.33 & 0.51 & 0.48 & 0.44 & 0.40 & 0.38 & 0.36 & 0.35 & 0.35 & 0.34 & 0.33 & 0.33 & 0.32 & 0.32 & 0.32 & 0.31 & 0.31 & 0.31 & 0.31 & 0.30 \\
\hline Min pulse width error & 0.22 & 0.22 & 0.22 & 0.22 & 0.22 & 0.21 & 0.21 & 0.21 & 0.22 & 0.22 & 0.22 & 0.22 & 0.22 & 0.23 & 0.21 & 0.23 & 0.23 & 0.23 & 0.22 & 0.22 \\
\hline Max pulse width error & 0.22 & 0.22 & 0.22 & 0.22 & 0.22 & 0.21 & 0.21 & 0.21 & 0.22 & 0.22 & 0.22 & 0.22 & 0.22 & 0.23 & 0.21 & 0.23 & 0.23 & 0.23 & 0.22 & 0.22 \\
\hline
\end{tabular}

\section{REFERENCES}

[1] R. Quijano Cetina, A. J. Roscoe, and P. S. Wright, "Challenges for Smart Electricity Meters due to Dynamic Power Quality Conditions of the Grid: A Review," AMPS 2017 - IEEE International Workshop on Applied Measurements for Power Systems, Proceedings, 2017.

[2] R. Masnicki, "Some Remarks on the Accuracy of Energy Meters," 2018 IEEE International Conference on Environment and Electrical Engineering and 2018 IEEE Industrial and Commercial Power Systems Europe (EEEIC / I\&CPS Europe), 2018.

[3] F. Leferink, C. Keyer, and A. Melentjev, "Static energy meter errors caused by conducted electromagnetic interference," IEEE Electromagnetic Compatibility Magazine, vol. 5, no. 4, pp. 49-55, Fourth 2016.

[4] J. Kirchhof and G. Klein, "EMV - Grenzwertlücke - Wechselrichter stört Zähler," 24. Symposium Photovoltaische Solarenergy, Bad Staffelstein, 2009.

[5] J. Kirchhof, "Grenzwertlücke - Wechselrichter stört Elektrizitätszähler," EMV, Düsseldorf, 2010.

[6] B. Have, T. Hartman, N. Moonen, C. Keyer, and F. Leferink, "Faulty Readings of Static Energy Meters Caused by Conducted Electromagnetic
Interference from a Water Pump," Submitted to Renewable Energy and Power Quality Journal (RE\&PQJ), 2019.

[7] "IEC 62053-21:2003 - Electricity metering equipment (a.c.) - particular requirements - part 21: Static meters for active energy (classes 1 and 2)."

[8] P. S. Wright, G. Rietveld, F. Leferink, H. E. V. den Brom, F. R. I. Alonso, J. P. Braun, K. Ellingsberg, M. Pous, and M. Svoboda, "Evaluation of EMI Effects on Static Electricity Meters," in 2018 Conference on Precision Electromagnetic Measurements (CPEM 2018), July 2018, pp. $1-2$.

[9] B. Have, T. Hartman, N. Moonen, and F. Leferink, "Inclination of Fast Changing Currents Effect the Readings of Static Energy Meters," Submitted to International Symposium on Electromagnetic Compatibility - EMC EUROPE, 2019.

[10] Chroma System Solutions, "Programmable AC/DC Electronic Load Model 63800 Series." [Online]. Available: http://www.chromausa.com/pdf/Br-63800-dcload-052014.pdf

[11] NH Research, "4600 Series Programmable AC Electronic Load." [Online]. Available: https://nhresearch.com/wp-content/uploads/datasheet-programmable-ac-electronic-load-4600-series.pdf 